\title{
Orange: Research analysis for wine study
}

\author{
Nikhil Gupta, Soham Trivedi, Hipal Gaudani, Mayank Gupta, Prasad Patil, Girish Gupta, \\ Vamsi Krishna K \\ Department of Biotechnology, Padmashree Dr. D.Y. Patil University, Navi Mumbai, 400614, India
}

\begin{abstract}
Fermentation of citrus juice may offer a relative simple avenue for reducing post harvest wastage of citrus fruits in low utilization environment and in places where the production of citrus concentrates is low or nonexistent. Orange juice concentrates are readily storable and can be used for production processes even when the fruit is out of season and investigated the possibility of exploiting the fermentative ability of yeasts to produce orange wines.
\end{abstract}

\section{Introduction}

Tropical citrus fruits have been used as substrates for the production of wines and the alcohol profiles, types and quantities were not stated in the majority of these reports. Various factors influence the fermentation process and determine the end products obtained. These include substrate related factors such as cultivar types, cultivation conditions, conditions at harvest and post harvest handling [1-11]. Yeast species are used in many industrial fermentation processes including alcoholic beverages production. The quality of wine produced greatly depends on the yeast strain [12]. Development of improved starter organisms for fermentation of citrus juice may offer a relative simple avenue for reducing post harvest wastage of citrus fruits in low utilization environment and in places where the production of citrus concentrates is low or nonexistent. Juice concentrates are readily storable and can be used for production processes even when the fruit is out of season $[13,14]$. In an effort aimed at increasing the low industrial utilization and reducing the high wastage of orange (Citrus sinensis) fruits in the developing world, and investigated the possibility of exploiting the fermentative ability of yeasts to produce orange wines. The types and quantities of alcohol present in orange wines produced from musts fermented by four strains of Saccharomyces species were determined. Citrus is the most economically important tree fruit crop in the world. The majority of citrus arrives to market in the form of processed products, such as single strength orange juice and frozen juice concentrates. In Florida, one of the world's top producing regions, more than $96 \%$ of all oranges are processed in too range juice.Inthe1999-2000 season, Florida produced more than four billion liters of single strength orange juice and as such, the Florida citrus juice industry is considered an important food processing industry in the United States. Economic losses due to juice spoilage are minimized by good sanitation procedures before and during citrus processing. Pasteurization, concentration, or low temperature storage protocols help to reduce the number of microorganisms in the final product. However, these products are not free of microbiological spoilage problems, especially non pasteurized single-strength juices. Citrus juices are acidic beverages (ca.pH3to4) with high Sugar content (15 ${ }^{\circ}$ Brix). Under these conditions, acidolactic bacteria, molds, and yeasts comprise the typical microbiota Present In citrus juices [15]. Potentiality of Rhizopus Oryzae to utilize orange peels under solid state fermentation conditions to produce macerating fluid with high cellulolytic and pectinolytic activities were confirmed in this work [16]. Yeast fermentation of orange juice shows at once, which has been no destructive effect on vitamin $\mathrm{C}$-a result in harmony with the observations, the contrary, the activity of vitamin $\mathrm{C}$ persisted for a very longtime-being retained for 51 days [17].

\section{History}

Chemical analyses of ancient organics absorbed into pottery jars from the early Neolithic village of Jiahu in Henan province in China have revealed that a mixed fermented beverage of rice, honey, and fruit (hawthorn fruit and/or grape, orange) was being produced as early as the seventh millennium before Christ (B.C.) [18]. The knowledge of wine and grapes as old as the cultural history of human population; the small consumption of wine could be advantageous. Also it has been well established for several thousands of years, that it could cause acute and chronic injuries after higher consumption. Its antiinfectious effect in dermatological drugs had been known in the archeological ages. The unguents containing wine polyphenols are advantageous in some dermatological injuries and also in the treatment of muscular and articular alterations [19]. Wine has been part of human culture for 6,000 years, serving dietary and socio-religious functions. Its production takes place on every continent, and its chemical composition is profoundly influenced by enological techniques. In addition to ethanol, which in moderate consumption can reduce mortality from coronary heart disease by increasing high-density lipoprotein cholesterol and inhibiting platelet aggregation, wine (especially red wine) contains a range of 
polyphenols that have desirable biological properties. These include the phenolic acids ( $p$ coumaric, cinnamic, caffeic, gentisic, ferulic, and vanillic acids), trihydroxy stilbenes (resveratrol and polydatin), and flavonoids (catechin, epicatechin and quercetin) [20].

\section{Analytical Technologies}

Polymerase Chain Reaction (PCR)

Polymerase chain reaction (PCR) identifies mixed-contaminated samples; identification of both species was hampered by preferential appearance of the melting peak coinciding with $H$. uvarum, except when $S$. cerevisiae was the dominating species. Application of whole genome amplification did not prevent the preferential detection of $H$. uvarum. This handicap was resolved by applying an enrichment procedure up to saturation after which the melting peak of both species could clearly be identified [21]. Because the yeast Brettanomyces produces volatile phenols and acetic acid, it is responsible for wine spoilage. The method was evaluated by an intralaboratory study for its specificity, linearity, repeatability and reproducibility. the standard curve was established from 14 different wines artificially inoculated. The quantification limit was $31 \mathrm{cfu} / \mathrm{mL}$ [22]. The main objective of this study was to select different strains of Brettanomyces bruxellensis isolated from red wines and to compare their volatile phenol production. From a collection of 63 strains, eight strains of $B$. bruxellensis were selected for volatile phenol production after the application of molecular techniques such as ISS-PCR, PCR-DGGE and REA-PFGE [23]. PCR method of direct DNA isolation from the wine microflora using polyvinylpyrrolidone in order to decrease the polyphenolic concentration proved to be efficient for the early and sensitive detection of ropy Ped. damnosus strains during wine-making [24]. The wine associated $A A B$ species, the aroma and flavour changes in wine due to $A A B$ metabolism, discusses the importance of oxygen ingress into the bottle and presents a hypothesis for the mechanism of spoilage of bottled red wine [25].

\section{Real-Time Polymerase Chain Reaction (RT- PCR)}

Real-time or quantitative PCR (QPCR) is developed for the rapid quantification of two of the most important yeast groups in alcoholic fermentation (Saccharomyces spp. and Hanseniaspora spp). The design of new primers for other important wine yeast species will enable to monitor yeast diversity during industrial wine fermentation and to detect the main spoilage yeasts in wine [26]. The objective of research was to develop a rapid, specific, and sensitive real-time PCR method for detecting spoilage molds during screening of raw materials and final product quality control analysis. With this set of primers and probe, less than 1,000 mold cells per milliliter of orange juice (10 cells per reaction) were detected with the real-time PCR system within 6 to $7 \mathrm{~h}$ [27]. This research work developed a quantitative real-time PCR (QPCR) for the rapid detection and quantification of $S$. cerevisiae in wine. The QPCR is highly reproducible, allowing the specific detection and quantification of this yeast in artificially contaminated wines, with a detection limit of 78 $\mathrm{CFU} / \mathrm{mL}$ [28]. This method detected yeast Brettanomyces produces volatile phenols and acetic acid; it is responsible for wine spoilage [22]. Quantitative real-time PCR assay for the rapid detection of $Z$. bailii from fruit juices and wine even in the presence of non-target DNA and assay provides a rapid and accurate method to establish the levels of the total $Z$. bailii population which consists of both viable and nonviable cells [29].

\section{Mass Spectometry (MS)}

Collaborative study of the carbon-13 isotope ratio mass spectrometry (13C-IRMS) method based on fermentation ethanol for detecting some sugar additions in fruit juices and maple syrup is reported. This method is complementary to the site-specific natural isotope fractionation by nuclear magnetic resonance (SNIF-NMR) method for detecting added beet sugar in the same products and uses the same initial steps to recover pure ethanol. The precision of this method for measuring delta13C was similar to that of other methods applied to wine ethanol or extracted sugars in juices [30]. Analysis covers two important techniques, high resolution nuclear magnetic resonance (NMR) spectroscopy and mass spectrometry (MS); used to characterize food products and detect possible adulteration of wine, fruit juices, and olive oil, all important products of the Mediterranean Basin [31].

\section{Gas Chromatography (GC)}

Gas Chromatography-MS and GC-flame ionization detection (FID)/olfactometry, 95 volatile components are detected in orange essence oil, of which 55 were aroma active. Over 20 compounds were identified for the first time in orange essence oil using MS; also, most did not exhibit aroma activity [32]. Gas chromatographymass spectrometry (GC-MS) and gas chromatography-olfactometry (GC-O) were used to determine the aromatic composition and aroma active compounds of fruit juice and peel oil of Jinchen sweet orange fruit. Nineteen components have been perceived only in the juice and ten compounds were described as aromatic components of only the peel oil by the panelists. These differences lead to the different overall aroma between fruit juice and peel oil [33]. Aromatic extract obtained by liquid-liquid extraction was representative of orange wine 
odor. Total of 63 compounds were identified and quantified in orange wine. Alcohols followed by terpenes and esters were the most abundant aroma-active compounds of the orange wine [34]. $\mathrm{GC} / \mathrm{O}$ analysis of canned lychees indicated that cis-rose oxide, linalool, ethyl isohexanoate, geraniol, furaneol, vanillin, (E)-2-nonenal, betadamascenone, isovaleric acid, and (E)-furan linalool oxide were the most odor potent compounds detected in the fruit extracts. While differences exist in the aroma profile of lychee and Gewürztraminer, the common odorants detected in both fruit and wine, particularly cisrose oxide are responsible for the lychee aroma in Gewürztraminer wine [35]. Method examines analytical findings, odor activity, and variations due to cultivar, sampling methods, manner of juicing, plus possible enzymatic and microbial artifacts. Initial attempts to characterize orange juice odor were based on volatile quantitation and overemphasized the importance of high concentration volatiles. Orange juice odor models confirm that fresh orange aroma is complex as the most successful models contain 23 odorants [36]. Gas liquid chromatography was used to identify the types of alcohol present in wines produced by fermenting orange juice with four strains of yeast; Saccharomyces cerevisiae (isolated from yam), $S$. cerevisiae (from sugarcane molasses), Saccharomyces carlsbergensis (from sugarcane molasses) and $S$. cerevisiae var. ellipsoideus (from orange juice) [5].

\section{Capillary Zone Electrophoresis}

Capillary zone electrophoresis given the analysis of inorganic and organic acid anions in orange juice and wine samples; CZE procedure provided a sensitive and economic technique for simultaneous determination of inorganic and organic acid anions in orange juice, red and white wine samples [37]. The application of electrophoresis to the determination of inorganic anions (sulphate, sulphite, phosphate, nitrate, nitrite and halides) and inorganic cations (ammonium, alkali and alkaline metals and trace elements) in food and beverages is presented [38]. The separation and detection of commonly used preservatives (benzoate, sorbate) and vitamin $\mathrm{C}$ by both conventional $\mathrm{CE}$ and microchip electrophoresis with capacitively coupled contact less conductivity detection are presented. This methods developed, are useful for the quantitative determination of food additives in real samples such as soft drinks and vitamin C tablets [39].

Effect of bottle colour and storage conditions New method investigated the effect of bottle colour, storage temperature and storage time on the browning of orange wine. The use of brown bottles and the short-time storage reduced the browning in orange wines; however, storage at two different temperatures did not significantly affect the browning index [40]. Experiments of accelerated oxidation of "fino" sherry wines have been conducted at 25 degrees $\mathrm{C}$ and under the influence of UV-visible radiation (a xenon lamp of $1500 \mathrm{~W})$. All wines showed losses in several polyphenolic compounds, which were more severe for the wines bottled in transparent glasses. Also, these wines exhibited a lower degree of visual browning (abs $420 \mathrm{~nm}$ ). In the research of volatile compounds, most of these presented increases during storage exposed to the influence of the UV-vis radiation [41].

\section{Evaluation of aroma differences}

Twenty-five odor-active compounds are quantified in hand-squeezed juices of Valencia late and Navel oranges using stable isotope dilution assays. Odor activity values (OAVs, ratio of the concentration to odor thresholds) based on odor thresholds in water were calculated for the entire set of aroma compounds in both varieties. High OAVs, the fruity-smelling esters ethyl 2methylpropanoate, ethyl butanoate, (S)-ethyl 2methylbutanoate, and 3a,4,5,7a-tetrahydro-3,6dimethyl-2(3H)-benzofuranone (wine lactone), the grassy smelling (Z)-hex-3-enal, and the citrus-like decanal are the most potent odorants in both juices. The weaker fruity note in the Navel oranges was clearly correlated with significantly lower OAVs of all fruity-smelling esters but a higher OAV of (Z)-3-hexenal compared to Valencia late. Model solutions simulating the odor of both orange varieties confirmed the findings of the quantitation studies [42].

\section{Effect of fermentation variables}

Sixteen yeast strains isolated from grapefruit (Citrus paradis), orange (Citrus sinensis) and pineapple (Ananas comosus) are characterized using standard microbiological procedures. The species are identified as Saccharomyces uvarum, S. cerevisiae, S. carlbergensis, and $S$. ellipsoideus. Their abilities for wine production are tested by using sugar and ethanol tolerance tests. The best biochemically active strain, $S$. ellipsoideus, is used along with commercially available baker's yeast ( $S$. cerevisiae) to produce wine from grapefruit, orange, and pineapple juices. After fermentation for $14 \mathrm{~d}$ with $S$. cerevisiae and $21 \mathrm{~d}$ with $S$. ellipsoideus, wines produced are compared with Baron de Valls (standard). The highest $(10.47 \%$ [v/v]) and lowest $(7.68 \% \quad[\mathrm{v} / \mathrm{v}])$ alcohol concentrations with corresponding residual sugar concentrations of $1.88 \%(\mathrm{w} / \mathrm{v})$ and $7.7 \%(\mathrm{w} / \mathrm{v})$ are produced from orange after fermentation with $S$. cerevisiae and $S$. ellipsoideus, respectively. $S$. ellipsoideus is found to be the best yeast strain producing wine with the highest acceptable score of 7.41 from orange. The study revealed the possibility of 
producing wine from our locally available fruit using simple, cheap, and adaptable technology with biochemically characterized yeast strains [43].

\section{in vitro, ex vivo, and in vivo antioxidant properties}

Grape juice is a powerful in vivo antioxidant, and this property, in combination with its platelet aggregation inhibition ability, can potentially reduce the risk of heart disease [44]. Grape juice, but not orange juice or grapefruit juice, inhibits human platelet aggregation. Coronary artery disease is responsible for much mortality and morbidity around the world. Platelets are involved in atherosclerotic disease development and the reduction of platelet activity by medications reduces the incidence and severity of disease. Red wine and grapes contain polyphenolic compounds, including flavonoids, which can reduce platelet aggregation and have been associated with lower rates of cardiovascular disease. Citrus fruits contain different classes of polyphenolics, which may not share the same properties. This study evaluated whether commercial grape, orange and grapefruit juices, taken daily, reduce ex vivo platelet activity. In a randomized cross-over design, ten healthy human subjects (ages 26-58 y, five of each gender) drank 5-7.5 mL/ (kg. d) of purple grape juice, orange juice or grapefruit juice for 7-10 d each. Platelet aggregation whole blood impedance aggregometry, Chronolog Model \#590 at baseline was compared to results after consumption of each juice. Drinking purple grape juice for one week reduced the whole blood platelet aggregation response to $1 \mathrm{mg} / \mathrm{L}$ of collagen by $77 \%$. Orange juice and grapefruit juice had no effect on platelet aggregation. The purple grape juice had approximately three times the total polyphenolic concentration of the citrus juices and was a potent platelet inhibitor in healthy subjects while the citrus juices showed no effect. The platelet inhibitory effect of the flavonoids in grape juice may decrease the risk of coronary thrombosis and myocardial infarction [45].

\section{Social drinking environment with food consumption}

Thirteen healthy subjects i.e. seven men and six women participated in three drinking studies to examine the maximum increase of breath alcohol concentration $(\mathrm{Br} A C)$ and the time taken to reach the maximum BrAC (peak) after a subject finished a 'one for the road' drink in a social environment with food consumption. They consumed hard liquor in the first study, beer in the second and wine in the third. Each study consisted of two days. Day 1 served as a control in which the subjects were monitored by breath tests to establish their basic BrAC curves. Day 2 was a repeat of Day 1 with the addition of the final drink 'for the road' consumed by the subjects once their BrAC started to decline. The average increase in BrAC was $0.017+/-0.009 \%$ for hard liquor, $0.016+/-0.008 \%$ for beer, and $0.016+/$ $0.007 \%$ for wine. The average time to peak was $18+/-8$ minutes for hard liquor, $16+/-2$ minutes for beer, and $20+/-10$ minutes for wine. The BrAC increase and the time to peak seemed to be independent of the type of alcoholic beverage consumed [46].

\section{Formation of urethan in treated beverages}

Isotope dilution analyses with tritium-labeled diethyl pyrocarbonate show that the carcinogen urethan is formed in orange juice, white wine, and beer. Commercial use of the antimicrobial food additive diethyl pyrocarbonate can result in urethan concentrations of 0.1 to 0.2 milligram per liter in orange juice and of the order of 1 milligram per liter in white wine and beer [47].

\section{Current research in orange and its application in wine}

Spontaneous fermentation and inoculated fermentation on the volatile compounds of orange wine is studied. Total of 17 and 19 volatile compounds were identified and quantified in spontaneous and inoculated fermentations, respectively. Esters followed by alcohols and terpenes were the most abundant volatile compounds in the two orange wines [48]. The aroma-active compounds of Pontianak orange peel oil (Citrus nobilis Lour. var. microcarpa Hassk.) were characterized by using gas chromatography-olfactometry (GC-O) and aroma extract dilution analysis (AEDA) techniques. Forty-one compounds were found to be aromaactive, which were mainly dominated by saturated and unsaturated aldehydes [49]. The thermal degradation of cyanidin 3-(6"-malonyl) glucoside and cyanidin 3-glucoside, which are the two major anthocyanins in blood orange juice, have been investigated using the $\mathrm{pH}$-differential method and RP-HPLC method [50].

\section{Conclusion}

Fermentation of the orange juice by $S$. cerevisiae from yam resulted in products with different concentrations of alcohol types despite the fact that the fermenting organisms are of the same species, it is therefore concluded that the source of the yeast is thus an important factor in the determination of the amount and types of fuel oils present in wines and hence the quality of the wine. Most potent volatile flavour aroma compounds of the orange wine were ethyl hexanoate, ethyl octanoate, linalool, citronellol, 2phenylethanol and eugenol were found, gives good flavour wine. It is good source of vitamin C and antioxidant had no effect of fermentation, it retained for long time. Orange fruits are cheap 
and large quantity fruits available in market use successfully for making wine; in future, orange wine emerging as new industry.

\section{References}

[1] Maldonado O., Rolz C. and Schneider deCabrera S. (1975) J. Food Sci. 40: 262-327.

[2] Anuna M.I., Sokari T.G., Akpapunam M.A. (1990) Discov. Innov. 2(2): 8-84.

[3] Ndip R.N., Akoachere J.F., Dopgima L.L., Ndip L.M. (2001) Appl. Biochem. Biotechnol. 95(3): 209-220.

[4] Osho A. (2005) Afr. J. Biotechnol. 4(7): 660662.

[5] Okunowo W.O., Okotore R.O., Osuntoki A.A. (2005) Afr. J. Biotechnol. 4 (11): 1290-1296.

[6] Daudt C.E., Ough C.S. (1973) Am. J. Enol. Vitic. 24(3): 130-135.

[7] Bell A.A., Ough C.S., Kliewer M.W. (1979) Thompson Seedless Grapevines. Am. J. Enol. Vitic. 30(2): 124-129.

[8] Liu S.Q. (2002) J. Appl. Microbiol. 92: 589601.

[9] Jonathan D., Errol W.H. (2000) N. Z. J. Crop Hortic. Sci. 28: 155-173.

[10] Joshi V.K., Sandhu D.K. (2000) Microbiological Examination of Source of Fermentation and Ethanol Productivity of the Isolates. 29: 323-334.

[11] Kourkoutas Y., Kanellaki M., Koutinas A.A., Tzia C. (2005) J. Food Eng. 69: 115123.

[12] Kunkee R.E. (1984) Food Microbiol. 1: $317-$ 327.

[13] Ramachandra H.G.R., Arun H.K. (2005) J. Diary Sci. 85: 395-404.

[14] Siddiq M., Harte J., Dolan K., Khan A., Collins JK, Perkins Veazie PM (2006) International Food Technology Meeting Abstracts. p. 223.

[15] Covadonga R. Arias, Jacqueline K. Burns, Lorrie M. Friedrich, Renee M. Goodrich, and Mickey E. (2002) Parish Appl Environ Microbiol. 68(4): 1955-1961.

[16] Hamdy, H. S. (2005) Annals Microbiol. 55(3): 205-211

[17] Lepkovsky S. and Hart E. B. (1925) (From the Department of Agricultural Chemistry, University of Wisconsin, Madison.) E. G. Hastings AND W. C. Frazier. (1925) (From the Department of Agricultural Bacteriology, University of Wisconsin, Madison.)

[18] McGovern P.E., Mirzoian A., Hall G.R. (2009) Proc Natl Acad Sci US A. 106(18):7361-6.

[19] Fehér J., Lengyel G., Lugasi A. (2005) Orv Hetil. 146(52):2635-9
[20] Soleas G.J., Diamandis E.P., Goldberg D.M. (1997) J Clin Lab Anal. 11(5):287313

[21] Renard A., Gómez di Marco P., EgeaCortines M., Weiss J. (2008) Int J Food Microbiol. 126(1-2):195-201

[22] Tessonnière H., Vidal S., Barnavon L., Alexandre H., Remize F. (2009) Int $J$ Food Microbiol. 129(3):237-43.

[23] Oelofse A., Lonvaud-Funel A., du Toit M. (2009) Food Microbiol. 26(4):377-85.

[24] Gindreau E., Walling E., Lonvaud-Funel A. (2001) J App/ Microbio/ 90(4):535-42.

[25] Bartowsky E.J., Henschke P.A. (2008) Int J Food Microbiol. 125(1):60-70

[26] Hierro N., Esteve-Zarzoso B., Mas A., Guillamón J.M.( 2007) FEMS Yeast Res. 7(8):1340-9

[27] Wan K., Yousef A.E., Schwartz S.J., Wang H.H.(2006 ) J Food Prot 69(2):385-90.

[28] Salinas F., Garrido D., Ganga A., Veliz G., Martínez C.(2009) Food Microbiol. 26(3):328-32

[29] Rawsthorne H., Phister T.G. (2006) Int $J$ Food Microbiol. 112(1):1-7

[30] Jamin E., Martin F., Martin G.G. (2004) $J$ AOAC Int. 87(3):621-31.

[31] Ogrinc N., Kosir I.J., Spangenberg J.E., Kidric J. (2003) A review. Anal Bioanal Chem. 376(4):424-30.

[32] Högnadóttir A., Rouseff R.L. (2003) J Chromatogr A. 998(1-2):201-11.

[33] Qiao Y., Xie B.J., Zhang Y., Zhang Y., Fan G., Yao X.L., Pan S.Y. (2008) Molecules. 13(6):1333-44.

[34] Selli S., Canbas A., Varlet V., Kelebek H., Prost C., Serot T. (2008) J Agric Food Chem. 56(1):227-34.

[35] Ong P.K., Acree T.E. (1999) J Agric Food Chem. 47(2):665-70.

[36] Perez-Cacho P.R., Rouseff R.L. (2008) Crit Rev Food Sci Nutr. 48(7):681-95.

[37] Fung Y.S., Lau K.M. (2003) Electrophoresis. 24(18):3224-32.

[38] Sádecká J., Polonský J. (1999) J Chromatogr A. 834(1-2):401-17.

[39] Law W.S., Kubán P., Zhao J.H., Li S.F., Hauser P.C. (2005) Electrophoresis. 26(24):4648-55.

[40] Selli S., Canbaş A., and Unal U. (2002) Nahrung. 46(2):64-7

[41] Benítez P., Castro R., García Barroso C. (2003) J Agric Food Chem. 51(22):6482-7.

[42] Buettner A., Schieberle P. (2001) J Agric Food Chem. 49(5):2387-94

[43] Ndip R.N., Akoachere J.F., Dopgima L.L., Ndip L.M. (2001) Appl Biochem Biotechnol. 95(3):209-20

[44] Vinson J.A., Yang J., Proch J., Liang X. (2000) J Med Food. 3(4):167-71. 
[45] Keevil J.G., Osman H.E., Reed J.D., Folts JD (2000) J Nutr.130(1):53-6.

[46] Breen M.H., Dang Q.T., Jaing J.T., Boyd G.N. (1998) Med Sci Law. 38(1):62-9.

[47] Löfroth G., Gejvall T. (1971) Science 174(15):1248-50

[48] Gang Fan,Xiaoyun Xu ,Yu Qiao, Yongxia Xu ,Yun Zhang, Li and Siyi Pan (2009)
European Food Research and Technology, 228 (6) 849-856.

[49] Dharmawan J., Kasapis S., Sriramula P., Lear M.J., Curran P. (2009) J Agric Food Chem. 57(1):239-44.

[50] Cao S., Liu L., Pan S., Lu Q., Xu X. (2009) J Agric Food Chem. 57(1):245-9. 